\title{
First Maintenance Therapy for Chronic Obstructive Pulmonary Disease: Retrospective Analyses of US and UK Healthcare Databases
}

\author{
Chloe I. Bloom (D) · Jukka Montonen · Olaf Jöns · Elizabeth M. Garry • \\ Surya P. Bhatt
}

Received: September 22, 2021 / Accepted: November 22, 2021 / Published online: January 10, 2022

(C) The Author(s) 2022

\section{ABSTRACT}

Introduction: Inhaled corticosteroids (ICS) are often prescribed inappropriately alongside longacting bronchodilators for chronic obstructive pulmonary disease (COPD). We aimed to investigate if prescribing habits in the US and UK differ from recommendations for initiation of COPD maintenance therapy.

Methods: We used healthcare data from the US IBM $^{\circledR}$ MarketScan ${ }^{\circledR}$ and UK Clinical Practice Research Datalink databases to assess exacerbations and comorbidities in patients with COPD initiating first maintenance therapy (1MT) between 2015 and 2018. Patients with a recorded asthma diagnosis prior to initiation of $1 \mathrm{MT}$

Supplementary Information The online version contains supplementary material available at https:// doi.org/10.1007/s41030-021-00179-0.

\section{I. Bloom $(\bowtie)$}

Imperial College London, London, UK

e-mail: chloe.bloom06@imperial.ac.uk

J. Montonen · O. Jöns

Boehringer Ingelheim International $\mathrm{GmbH}$,

Ingelheim am Rhein, Germany

E. M. Garry

Aetion Inc., Boston, MA, USA

\section{S. P. Bhatt}

Division of Pulmonary, Allergy and Critical Care Medicine, University of Alabama at Birmingham, Birmingham, AL, USA were excluded. We evaluated time from recorded diagnosis of COPD until initiation of 1MT, and treatment regimen at 1MT (long-acting muscarinic antagonist [LAMA], long-acting $\beta_{2^{-}}$ agonist [LABA], ICS, as monotherapy or in combination).

Results: In the US and UK, median (IQR) time between recorded COPD diagnosis and 1MT was $158(12 ; 839)$ and $29(1 ; 521)$ days, respectively. Among the 53,473 US patients and 8786 UK patients who initiated $1 \mathrm{MT}, 50.9 \%$ and $32.4 \%$ had $\geq 1$ exacerbation in the previous year. In the US, $20 \%$ of patients initiated LAMA, $1 \%$ LABA, 13\% LAMA/LABA, and 66\% an ICS-containing regimen (49\% LABA/ICS, 13\% ICS, and $4 \%$ LAMA/LABA/ICS). In the UK, $53 \%$ of patients initiated LAMA, 4\% LABA, 16\% LAMA/ LABA, and $27 \%$ an ICS-containing regimen (14\% LABA/ICS, 9\% ICS, and 4\% LAMA/LABA/ ICS).

Conclusions: At 1MT, two-thirds of patients in the US received ICS-containing therapies, with almost half on LABA/ICS. In contrast, less than one-third received ICS-containing therapy in the UK and more than half of patients received LAMA. In both countries, more patients received ICS-containing therapies at initiation of 1MT than would be expected based on their exacerbation history, suggesting overprescribing. 


\section{PLAIN LANGUAGE SUMMARY}

Chronic obstructive pulmonary disease (COPD), a smoking-related lung disease, restricts airflow in the lungs, causing symptoms such as breathlessness and coughing. To control symptoms, patients use one or more types of inhaled 'maintenance' medication, which can be prescribed alone or together. When patients have a short-term worsening of symptoms, doctors often prescribe inhaled corticosteroids (ICS). We wanted to see whether doctors' prescribing habits for maintenance inhalers are in line with clinical guidelines, so we analyzed data from a US health insurance database $(\sim 50,000$ patients) and UK primary care medical records ( $\sim 8000$ patients). We focused on patients with a diagnosis of COPD who were prescribed their first maintenance therapy (1MT) between 2015 and 2018. We excluded people with a diagnosis of both COPD and asthma, as similar inhalers are used to treat these conditions, although the clinical decisions for when to prescribe them differ. The average time between COPD diagnosis and 1MT was longer in the US (158 days) than in the UK (29 days). A higher percentage of patients in the US $(\sim 65 \%)$ versus UK $(\sim 25 \%)$ were prescribed ICS as part of their treatment, and ICS use in both countries was higher than expected based on the guidelines, which recommend ICS only for patients with severe COPD who meet certain criteria. Our findings suggest overprescribing of ICS in both countries (particularly the US), meaning that some patients are being given medication without a known clinical benefit, which puts them at risk of side effects, possibly increasing unnecessary healthcare costs.

Keywords: Bronchodilators; Clinical guidelines; COPD; Database; Inhaled corticosteroid; Long-acting beta-agonists; Long-acting muscarinic antagonists; Maintenance therapy; Observational study

\section{Key Summary Points}

We analyzed $\sim 53,000$ patients from a large commercial US healthcare database and $~ 9000$ patients from a UK primary care database who had initiated their first maintenance therapy (1MT) for COPD between 2015 and 2018 .

The time gap between diagnosis of COPD and initiation of $1 \mathrm{MT}$ was 158 days in the US and 29 days in the UK.

In the US cohort, in which $51 \%$ of patients had $\geq$ acute exacerbation in the year prior to $1 \mathrm{MT}$, the most common 1MT was a long-acting $\beta_{2}$-agonist combined with inhaled corticosteroids (ICS) (49\%).

In the UK cohort, in which $32 \%$ of patients had $\geq 1$ acute exacerbation in the year prior to $1 \mathrm{MT}$, the most common $1 \mathrm{MT}$ was a long-acting muscarinic antagonist monotherapy (53\%).

In the US and UK, $66 \%$ and $27 \%$ of patients were prescribed ICS-containing therapies as $1 \mathrm{MT}$, respectively. In both countries, ICS were overprescribed based on guideline recommendations regarding exacerbation history.

\section{INTRODUCTION}

Chronic obstructive pulmonary disease (COPD) is a major cause of morbidity and mortality worldwide, with significant medical and economic burden, both in terms of hospitalizations for the treatment of exacerbations as well as the costs of maintenance therapy and outpatient treatment [1]. COPD is the fourth-leading cause of death in the US and the fifth-leading in the UK $[2,3]$. An estimated 24 million adults in the US are living with COPD $[4,5]$, with more than USD 32 billion spent on COPD-related patient 
care in 2010 [6]. In the UK, an estimated 1.2 million people are living with diagnosed COPD [7], and the British Lung Foundation estimates that COPD costs the UK National Health Service GBP 1.9 billion annually [8, 9].

The goals of COPD treatment are to reduce symptoms, decrease the risk of exacerbations, and improve health status and exercise tolerance [10]. To achieve these goals, recommendations from the Global Initiative for Chronic Obstructive Lung Disease (GOLD) 2021 strategy report offer a model for initiation of pharmacotherapy, based on an individualized assessment of symptoms and exacerbation risk (the ABCD classification system), as well as the presence of comorbidities (Supplementary Table 1) [10-12]. Long-acting bronchodilators, either as monotherapy (long-acting muscarinic antagonist [LAMA] or long-acting $\beta_{2}$-agonist [LABA]) or dual therapy (LAMA/LABA) [10] are the most common initial maintenance therapy for patients with COPD, with inhaled corticosteroids (ICS) reserved as an add-on therapy (in combination with one or two bronchodilators) for patients with a history of asthma, or a history of exacerbations ( $\geq 2$ moderate exacerbations or $\geq 1$ exacerbation leading to hospitalization in the previous year) together with a blood eosinophil count of $\geq 300$ cells $/ \mu$ l, for patients with one exacerbation in the previous year, or $\geq 100$ cells/ $\mu$ l, for patients with $\geq 2$ moderate exacerbations or $\geq 1$ exacerbation leading to hospitalization in the previous year [10].

Guidelines from the American Thoracic Society (ATS) recommend LAMA/LABA over LAMA or LABA monotherapy in patients with COPD and dyspnea or exercise intolerance, with triple therapy (LAMA/LABA/ICS) only recommended for patients who have had $\geq 1$ exacerbation in the past year $[11,13]$. In the UK, the National Institute for Health and Care Excellence (NICE) recommends that patients are treated with a LAMA/LABA combination unless they have features suggesting asthma or steroid responsiveness, in which case they should be offered LABA/ICS [12].

Despite this guidance, several studies investigating prescribing patterns among patients with COPD in clinical practice suggest that ICS are often prescribed for less severe disease than is recommended [14-17], which can expose patients to the unnecessary risk of local adverse events (AEs) such as oral thrush and hoarseness; respiratory AEs such as pneumonia; and systemic AEs such as osteoporosis/risk of bone fractures, cataracts and glaucoma, diabetes and skin thinning/bruising [18-21]. Given these previously published reports, we aimed to further investigate if, and to what extent, prescribing habits in the US and UK differ from each other, and from global and national recommendations for initiation of maintenance therapy.

To do this, we conducted a study using two databases from the US and UK to assess exacerbations and comorbidities in patients with COPD without prior asthma, who initiated first maintenance therapy (1MT) between 2015 and 2018. We also aimed to evaluate the time from recorded diagnosis of COPD to initiation of $1 \mathrm{MT}$, and treatment choice at 1MT (LAMA, LABA, ICS monotherapy or combination).

\section{METHODS}

\section{Study Design and Patients}

This retrospective cohort study analyzed longitudinal electronic healthcare records from the US and the UK. Patients eligible for inclusion were at least 40 years old, with a confirmed COPD diagnosis (identified using International Classification of Diseases and Related Health Problems [ICD] codes in the US and Read codes in the UK; Supplementary Table 2) and $\geq$ 12 months of prior continuous enrollment in the US and UK databases in order to establish baseline characteristics.

Eligible patients had initiated maintenance therapy in the form of LAMA, LABA or ICS (mono therapy, dual therapy, or triple therapy) between 21 May 2015 and 31 March 2018 in the US (based on dispensing records), and between 1 July 2015 and 31 Dec 2018 in the UK (based on prescriptions). Patients were followed up until end of study period, end of continuous enrollment, or death. Patients diagnosed with asthma during the follow-up period were 
censored at the time of asthma diagnosis, and therefore not included in the analysis.

Maintenance therapy was defined as at least 30 days of continuous use of LAMA, LABA or ICS as monotherapy, dual therapy or triple therapy (open- or fixed-dose combination) after the recorded COPD diagnosis (first patient health claim for COPD [US]; first appearance of Read code in Clinical Practice Research Datalink [CPRD] database [UK]), including a 14-day allowable gap between individual drug fills to account for possible under-dosing of maintenance therapies and skipping or forgetting to take doses (Fig. 1).

As this was an observational study based on de-identified patient-level data from healthcare databases reported in aggregate only, the study did not require ethics committee approval, nor were subjects required to provide informed consent.

\section{The US Data Source}

The US data were from the IBM ${ }^{\circledR}$ MarketScan ${ }^{\circledR}$ database, which is drawn from large employers, health plans and public organizations in the US, including the Commercial Claims and Encounters Database, the Medicare Supplemental and Coordination of Benefits database, and the Medicaid database. The databases capture longitudinal, individual-level data on healthcare utilization, healthcare expenditures and plan enrollment, and contain integrated records for patient demographics, inpatient and outpatient events, and medicines dispensed by pharmacies from all US states.

\section{The UK Data Source}

The UK data were from the CPRD GOLD, an electronic database of de-identified longitudinal electronic health records from general practitioner (GP) practices across the UK [22]. The database includes diagnostic and prescribing information for each registered patient [22-24]; however, dispensing data are not recorded. For access to CPRD data, a protocol (number: 19_147) was approved by an Independent Scientific Advisory Committee.

\section{Study Variables}

We used ICD codes (US) and Read codes (UK) to describe the characteristics of patients with COPD according to various demographic and clinical characteristics (Supplementary Table 2), and concomitant medication use, at the date of initiation of any monotherapy, dual therapy or triple combination of LAMA, LABA or ICS as 1MT after 21 May 2015 (US) or 1 July 2015 (UK). We also estimated the time between COPD diagnosis and 1MT.

With the exception of comorbidities, all baseline variables were assessed in the 365-day baseline period before recorded COPD diagnosis. Chronic comorbidities were assessed between the earliest date available in the databases (2008 in the US and 1987 in the UK) and diagnosis of COPD. Ratio of forced expiratory volume in $1 \mathrm{~s}$ to forced vital capacity $\left(\mathrm{FEV}_{1} /\right.$ FVC; available in the UK database only) was reported based on the last available record prior to initiation of $1 \mathrm{MT}$.

The number of COPD exacerbations was estimated during the 365-day period prior to initiation of 1MT. COPD exacerbations in the US were defined as any of the following: an acute COPD exacerbation diagnosis; a claim for an oral antibiotic or an oral corticosteroid on the same day, or within 7 days following an outpatient COPD diagnosis/visit; an inpatient COPD diagnosis; hospitalization due to a respiratory condition; an emergency department visit with a COPD diagnosis; or a lower respiratory tract infection diagnosis [25]. In the UK, COPD exacerbations were defined as an acute COPD exacerbation or respiratory failure diagnosis; a lower respiratory tract infection diagnosis; or a prescription for an oral antibiotic or oral corticosteroid on the same day, or within 7 days following a COPD diagnosis (in line with a previous classification strategy proposed by Rothnie et al.) [26].

\section{Statistical Analyses}

Continuous variables were presented as mean (standard deviation) and/or median [interquartile range (IQR)], and categorical variables were 
A

1: LAMA drug use occurs continuously for 30 days

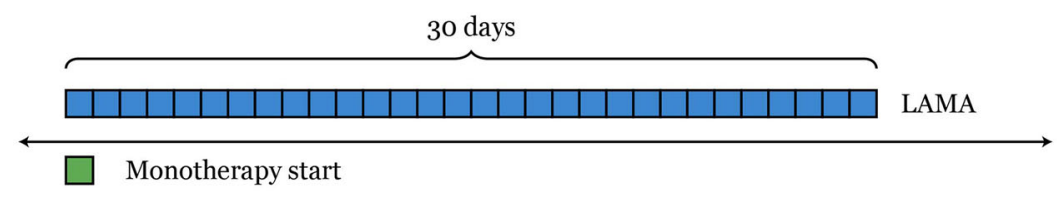

2: LAMA drug use occurs for multiple periods with a gap of 14 days or less

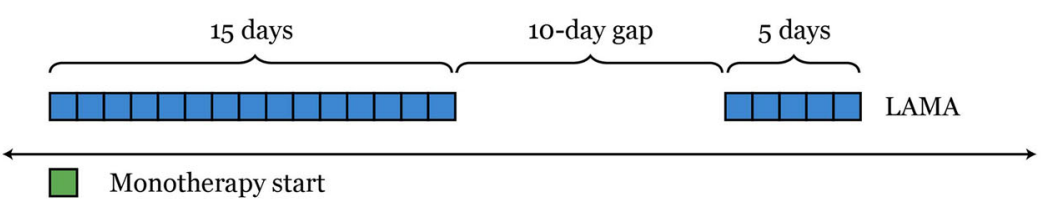

3: LAMA drug use occurs for multiple periods with a gap of 14 days or less, no overlap with other drugs for 30 days

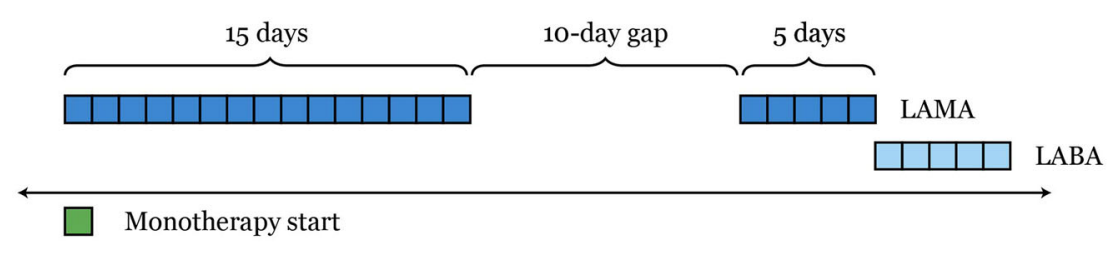

B

1: Continuous use of LAMA and LABA, overlapping for at least 30 days

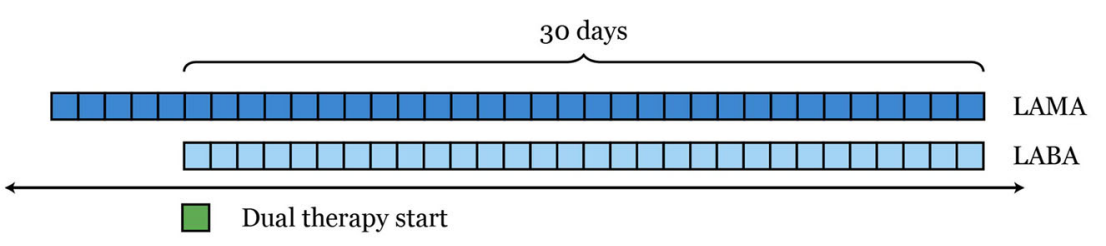

2: LAMA and LABA overlap for at least 30 days, with a 14-day allowable gap between individual drug fills

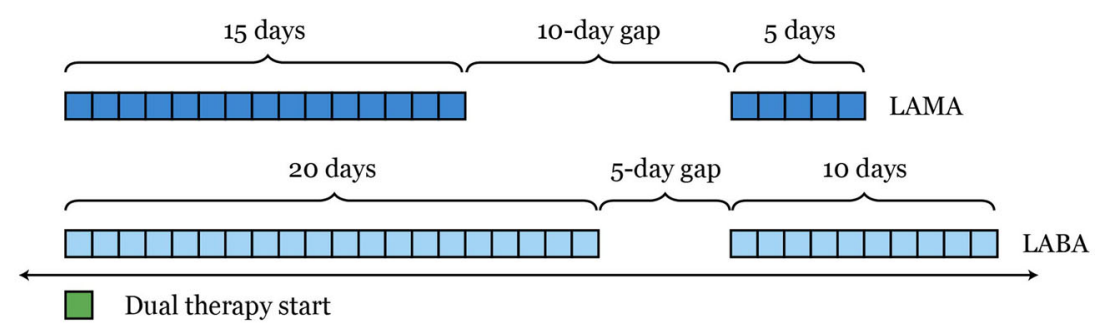

3: Continuous use of LAMA/LABA as a dual fixed-dose combination therapy (FDC), for at least 30 days with allowable gap

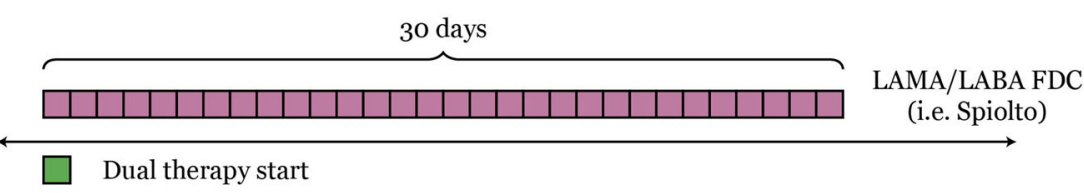

Fig. 1 Possible scenarios for maintenance therapy. A Monotherapy. B Dual therapy. C Triple therapy 


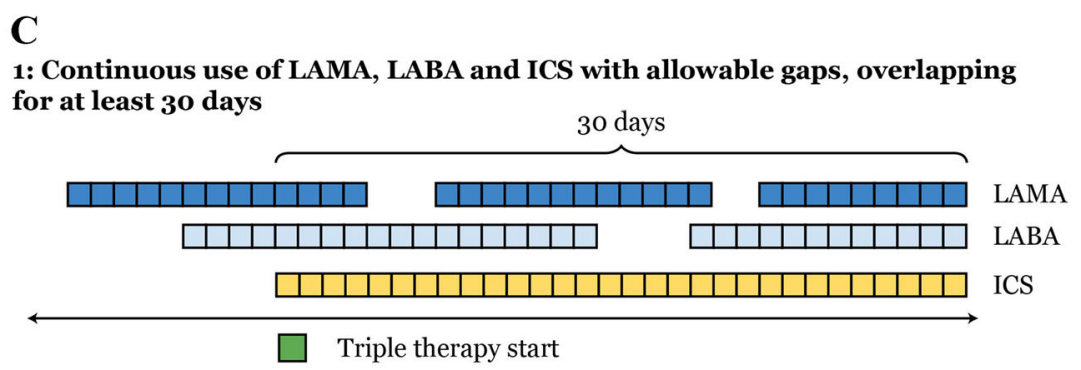

2: Continuous use of LAMA and LABA/ICS fixed-dose combination (FDC) with allowable gaps, overlapping for at least 30 days

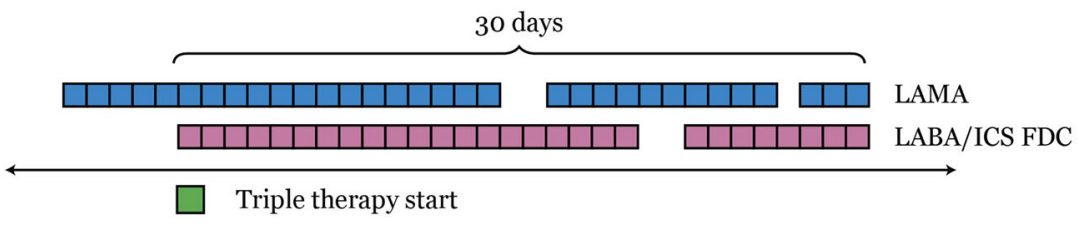

3: Continuous use of LAMA/LABA/ICS as a triple FDC therapy for at least 30 days with allowable gap

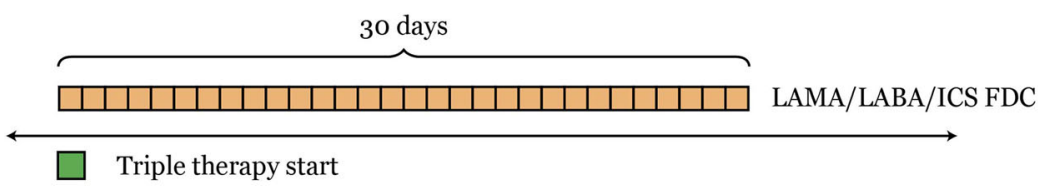

Fig. 1 continued

presented as absolute and relative frequencies. Data were analyed both overall and stratified by maintenance therapy regimen. The distribution of therapies used as 1MT was also described.

Missing data in covariates or descriptive variables were classified as their own category. In addition, a feasibility assessment was performed before beginning the analyses, and variables with $>75 \%$ missing values were excluded from all analyses, except baseline characteristics. If a variable was left with only one category other than that of missing, the variable was completely excluded.

All analyses were conducted using the Aetion Evidence Platform ${ }^{\circledR}$ (2020; Aetion Inc., New York, NY, USA), a software platform for realworld data analysis, which has been validated for a range of studies [27].

\section{RESULTS}

\section{Patient Characteristics at Initiation of First Maintenance Therapy (1MT)}

In total, 53,473 patients from the US and 8786 patients from the UK who initiated 1MT were included in the analysis (Fig. 2). Patient characteristics at 1MT are presented for the US (Table 1) and the UK (Table 2). Within each country, patient age and gender distribution at $1 \mathrm{MT}$ were similar across the treatment groups. $\mathrm{FEV}_{1} / \mathrm{FVC}$ data, which were only available in the UK, were also similar across treatment groups.

\section{Exacerbations}

Data for exacerbations are presented for the US (Table 1) and the UK (Table 2).

In the US, $50.9 \%$ of patients had $\geq 1$ exacerbation in the year preceding the initiation of 1MT (range: $43.4 \%$ [LAMA/LABA] to $69.8 \%$ 
A

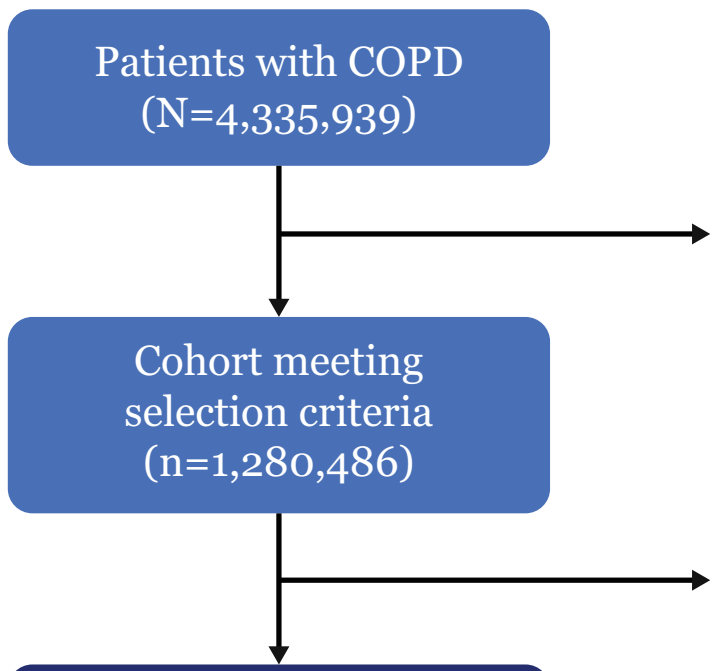

Patients initiating 1MT included in the analysis $(\mathrm{n}=53,473)$

B

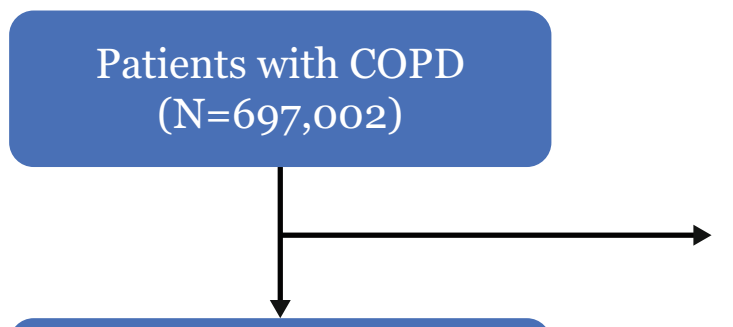

Cohort meeting selection criteria $(\mathrm{n}=91,855)$

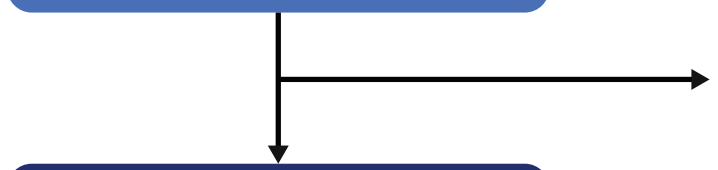

\section{Patients initiating 1MT included in the analysis $(n=8786)$}

Excluded due to $<365$ days continuous enrollment with medical and pharmacy benefit prior to COPD diagnosis, age $<40$ years

$$
(\mathrm{n}=3,055,453)
$$

Excluded due to 1MT initiation before 21st May 2015 or asthma diagnosis prior to index date

$$
(n=1,227,013)
$$

Excluded due to $<365$ days continuous enrollment prior to COPD diagnosis, age $<40$ years, or missing age or gender $(\mathrm{n}=605,147)$
Excluded due to 1MT initiation before 1st July 2015 or asthma diagnosis prior to index date $(\mathrm{n}=83,069)$

Fig. 2 Cohort selection criteria and patient counts. A US (MarketScani $\left.{ }^{\circledR}\right)$. B UK (CPRD). IMT first maintenance therapy; $C O P D$ chronic obstructive pulmonary disease 


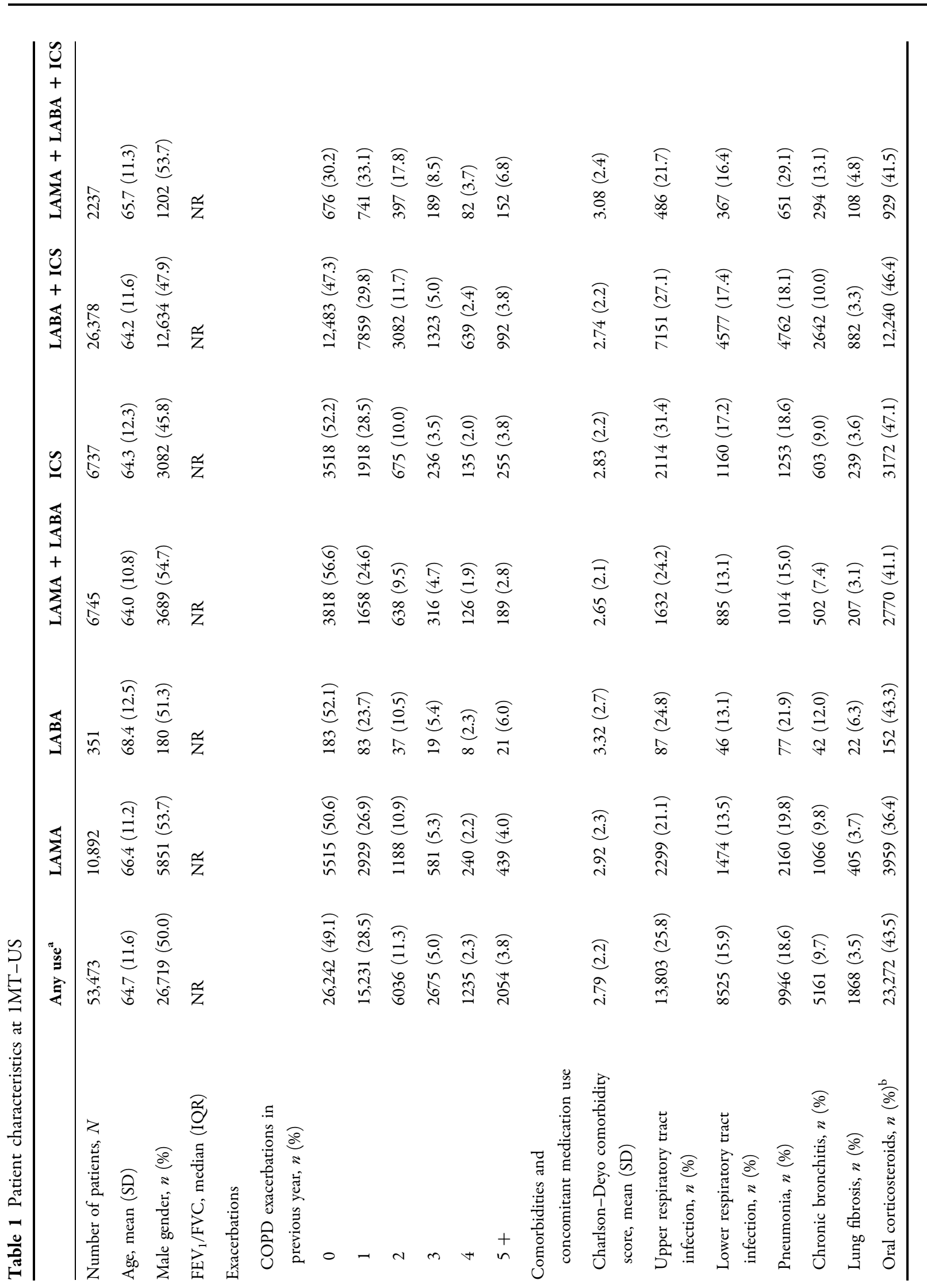




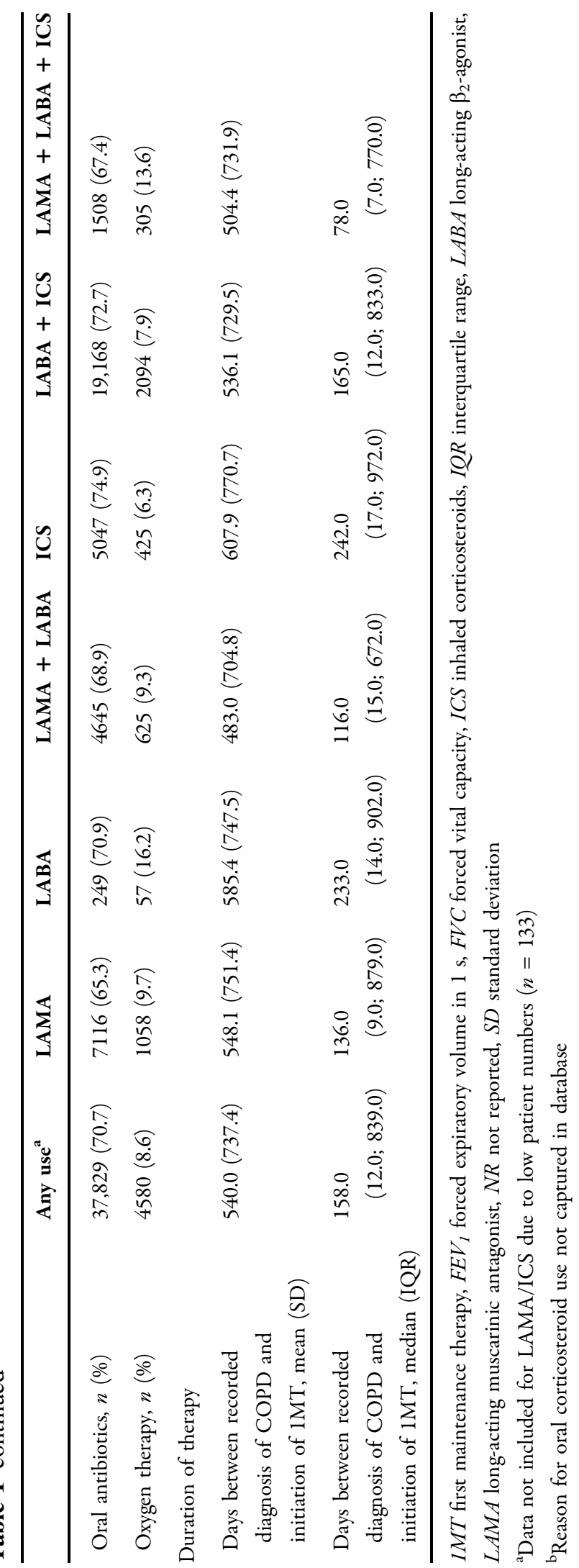




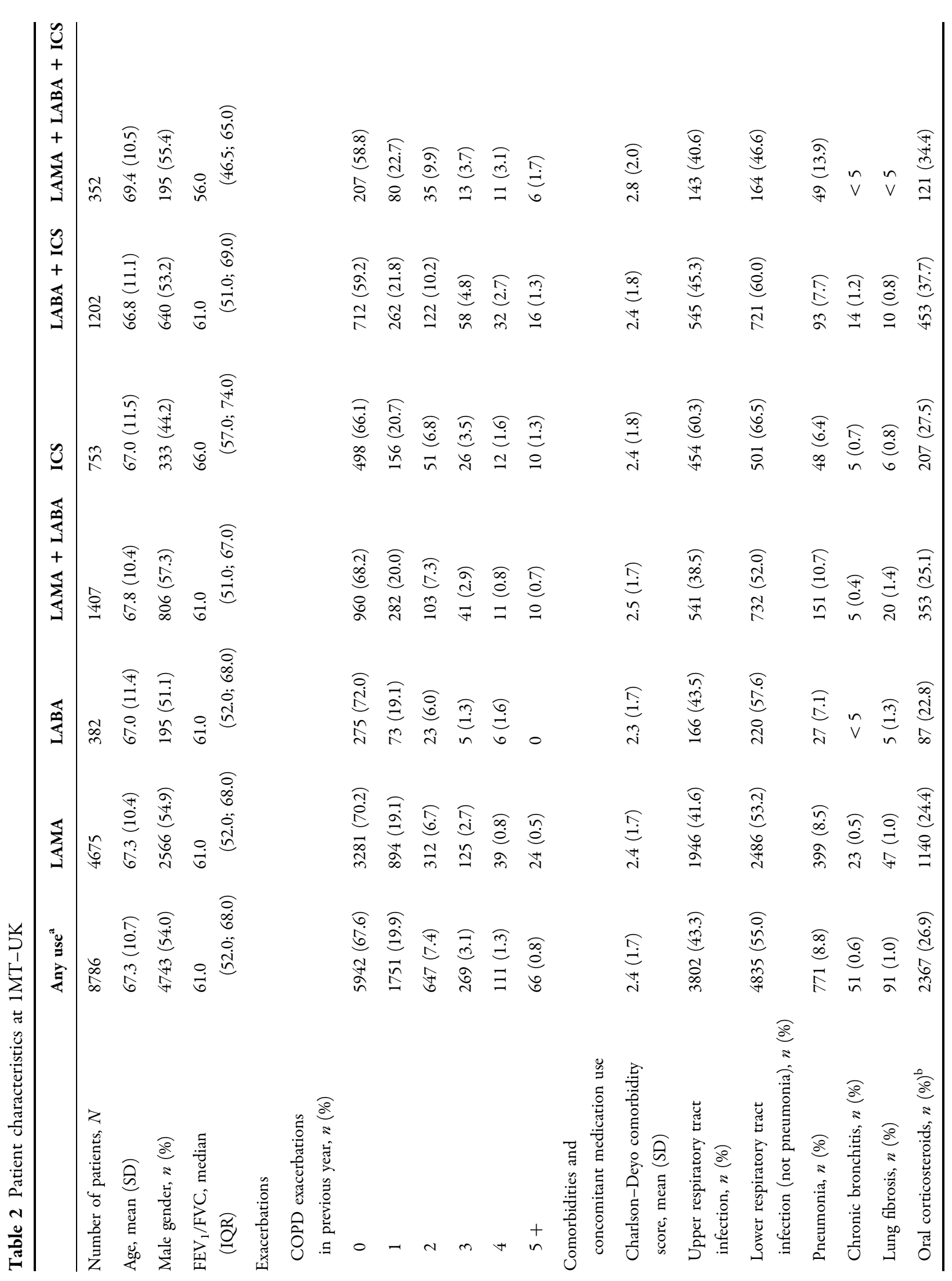




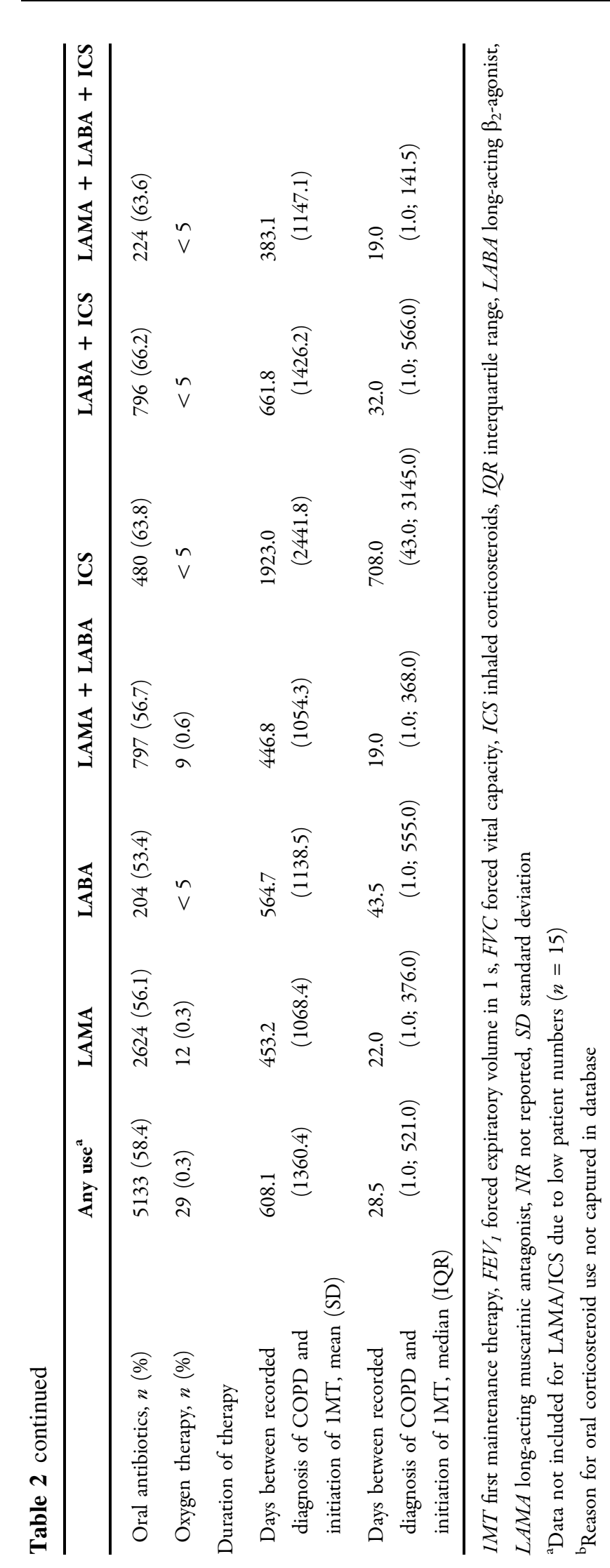


[LAMA/LABA/ICS]). When considering ICS users in the US (ICS, LABA/ICS, LAMA/ICS or LAMA/LABA/ICS), $47.1 \%$ of patients had no record of exacerbation in the year preceding the initiation of 1MT.

In the UK, $32.4 \%$ of patients had $\geq 1$ exacerbation in the year preceding the initiation of 1MT (range: $28.0 \%$ [LABA] to $41.2 \%$ [LAMA/ LABA/ICS]). When considering ICS users in the UK (ICS, LABA/ICS, LAMA/ICS or LAMA/LABA/ ICS), $61.4 \%$ of patients had no record of exacerbation in the year preceding the initiation of $1 \mathrm{MT}$.

\section{Respiratory Burden and Comorbidities}

In the US, $25.8 \%$ and $15.9 \%$ of patients had a recorded history of upper and lower respiratory tract infections at $1 \mathrm{MT}$, respectively. The percentage of patients with a history of pneumonia and chronic bronchitis at $1 \mathrm{MT}$ was $18.6 \%$ and $9.7 \%$, respectively. A history of lung fibrosis was reported in $3.5 \%$ of patients (Table 1 ).

In the UK, $43.3 \%$ and $55.0 \%$ of patients had a recorded history of upper and lower respiratory tract infections at $1 \mathrm{MT}$, respectively. The percentage of patients with a history of pneumonia and chronic bronchitis at $1 \mathrm{MT}$ was $8.8 \%$ and $0.6 \%$, respectively. A history of lung fibrosis was reported in $1.0 \%$ of patients (Table 2 ).

\section{Medication Use}

In the US, the percentage of patients using oral corticosteroids in the year preceding the initiation of $1 \mathrm{MT}$ was $43.5 \%$ (range: $36.4 \%$ [LAMA] to $47.1 \%$ [ICS]) and the percentage using oral antibiotics was $70.7 \%$ (range: $65.3 \%$ [LAMA] to $74.9 \%$ [ICS]). Use of oxygen therapy in the year prior to $1 \mathrm{MT}$ was reported by $8.6 \%$ of patients (range: $6.3 \%$ [ICS] to $16.2 \%$ [LABA]; Table 1).

In the UK, the percentage of patients using oral corticosteroids in the year preceding the initiation of $1 \mathrm{MT}$ was $26.9 \%$ (range: $24.4 \%$ [LAMA] to $37.7 \%$ [LABA/ICS]) and the percentage using oral antibiotics was $58.4 \%$ (range: $53.4 \%$ [LABA] to $66.2 \%$ [LABA/ICS]). Use of oxygen therapy in the year prior to $1 \mathrm{MT}$ was reported by $0.3 \%$ of patients (range: $0.3 \%$ [LAMA] to $0.6 \%$ [LAMA/LABA]; Table 2).

\section{Treatment Choice at 1MT}

In the US, $66.1 \%$ of patients initiated an ICScontaining regimen as $1 \mathrm{MT}$ (49.3\% LABA/ICS, $12.6 \%$ ICS, and $4.2 \%$ LAMA/LABA/ICS), followed by LAMA (20.4\%), LAMA/LABA (12.6\%), and LABA (0.7\%) (Fig. 3).

In the UK, $26.3 \%$ of patients initiated an ICScontaining regimen as 1MT $(13.7 \%$ LABA/ICS, $8.6 \% \mathrm{ICS}$, and $4.0 \%$ LAMA/LABA/ICS), with

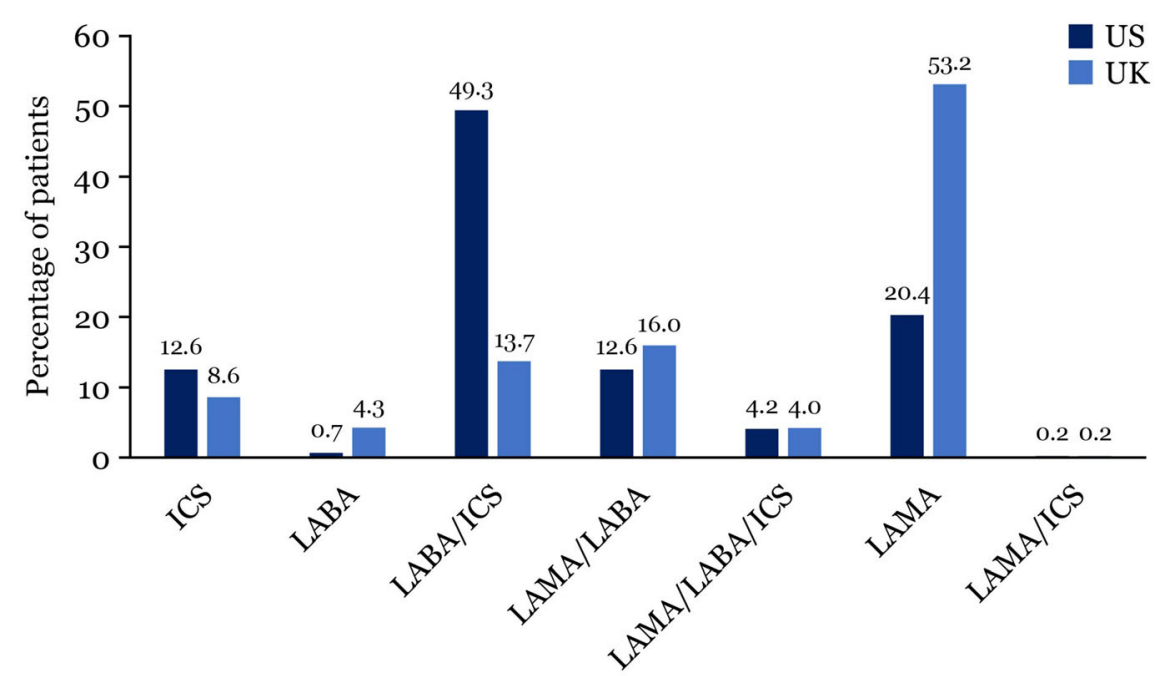

Fig. 3 Treatments received in the US and UK as $1 \mathrm{MT}^{\mathrm{a}}$. ${ }^{\text {a } T h e ~ c a t e g o r i z a t i o n ~ i s ~ m u t u a l l y ~ e x c l u s i v e ~ a n d ~ c o l l e c t i v e l y ~}$ exhaustive. IMT first maintenance therapy, CPRD Clinical Practice Research Datalink, GOLD Global Initiative for Chronic Obstructive Lung Disease, ICS inhaled corticosteroids, $L A B A$ long-acting $\beta_{2}$-agonist, $L A M A$ long-acting muscarinic antagonist 
LAMA the most common 1MT (53.2\%), followed by LAMA/LABA (16.0\%), and LABA (4.3\%) (Fig. 3).

\section{Time from Diagnosis to 1MT}

In the US, median (IQR) time from diagnosis to initiation of $1 \mathrm{MT}$ was $158(12 ; 839)$ days ranging from 78 days (LAMA/LABA/ICS) to 242 days (ICS; Table 1). In the UK, median (IQR) time from diagnosis to initiation of 1MT was 29 (1; 521) days ranging from 19 days (LAMA/LABA and LAMA/LABA/ICS) to 708 days (ICS; Table 2).

\section{DISCUSSION}

In this retrospective analysis of healthcare data from the US and UK, the overall patient populations were similar between the two countries in terms of age and gender distribution, but different in terms of disease severity. Patients in the US group had a higher exacerbation history than those in the UK group $(50.9 \%$ vs. $32.4 \%$ with $\geq 1$ exacerbation in the year prior to $1 \mathrm{MT}$ ), as well as a higher prevalence of comorbidities (with the exception of upper and lower respiratory tract infections) and concomitant medication use (oral corticosteroids and antibiotics). Comparison of treatment groups within each country showed that patient and disease characteristics were generally similar across the therapy classes in both the US and UK. The median time from diagnosis to initiation of 1MT was much longer in the US (158 days) compared with the UK, where patients initiated their 1MT on average within a month (29 days). Regarding prescribing patterns, striking differences were observed between the US and the UK, with almost half of patients (49\%) prescribed LABA/ICS at initiation of 1MT in the US, compared with over half (53\%) of patients prescribed LAMA monotherapy in the UK primary care setting at $1 \mathrm{MT}$.

The reason for the observed difference in time from diagnosis to initiation of $1 \mathrm{MT}$ between the US and UK is not clear, though several factors (in addition to the inherent differences in the data sources) may have contributed. In the US, the cost implications of initiating maintenance therapy (e.g., type of health plan coverage) may have delayed prescription claims, whereas this is unlikely to have been a factor in the UK, where the cost of prescription is the same regardless of inhaler (e.g., single versus dual) and free for those aged over 60 years. It is also possible that patients in the US had milder disease (e.g., they were diagnosed at an earlier stage of disease) than patients in the UK, and were therefore able to achieve adequate control with, for example, short-acting bronchodilators for symptom relief for a longer period before initiating long-acting maintenance therapy. However, in the absence of spirometry data from the US database, and quantitative dyspnea scores from both US and UK databases, it is not possible to state this with any degree of certainty.

At $1 \mathrm{MT}$ in the UK, $53 \%$ of patients were prescribed LAMA, consistent with the 2010-2018 NICE guidelines [28, 29], which recommend initiation of LAMA monotherapy in patients with breathlessness or exacerbations (32\% in this study). In the US, prescribing patterns reflect the ATS guidelines, which recommend ICS as an add-on therapy to long-acting bronchodilators in patients with a history of $\geq 1$ exacerbation in the past year who required antibiotics or oral steroids $[11,13]$. In our study, $49 \%$ of US patients received LABA/ICS as $1 \mathrm{MT}$, and $53 \%$ of these patients reported $\geq 1$ exacerbation in the year preceding initiation of $1 \mathrm{MT}$ (in total, $44 \%$ of patients had used oral corticosteroids and $71 \%$ had used oral antibiotics). These data suggest concordance between patients' exacerbation history and ATS guidelines for use of ICS-containing therapy. In the context of GOLD, which recommends addition of ICS to LABA in patients with $\geq 2$ moderate exacerbations (or 1 exacerbation leading to hospitalization) in the previous year, the US data suggest that ICS are overprescribed. Only $22 \%$ of US patients had $\geq 2$ exacerbations in the year prior to $1 \mathrm{MT}$, yet $66 \%$ of patients were prescribed ICS-containing treatments. This means that many of these prescriptions were not in accordance with GOLD recommendations in regard to history of previous exacerbations [10]. However, additional data would be 
required to make a fuller assessment of adherence to GOLD, including the severity of exacerbations and the percentage of patients with a blood eosinophil count $\geq 300$ cells $/ \mu$ l at $1 \mathrm{MT}$. Indeed, the absence of data on exacerbation severity in our study precludes any assessment of how many patients with 1 exacerbation in the previous year would have qualified for escalation to ICS based on the GOLD recommendations (had these patients required hospitalization for their exacerbation).

In the UK, $26 \%$ of patients were prescribed ICS-containing regimens at $1 \mathrm{MT}$, with $12 \%$ experiencing $\geq 2$ exacerbations in the previous year. Hence, similar to the US cohort, many patients may have been overprescribed ICS, based on their exacerbation history and the GOLD and NICE guidelines, considering that patients with a co-diagnosis of asthma were excluded from the analysis [12] (the 2010-2018 NICE guidelines state that triple therapy should be considered in the presence of persistent symptoms or exacerbations despite bronchodilator monotherapy, but do not quantify the term 'persistent').

In the accompanying paper in this issue, we report that, based on exacerbation history, ICS is also overused as second maintenance therapy (2MT) compared with global and national recommendations. At 2MT, around one in three patients in both the US and UK were prescribed triple therapy (LAMA/LABA/ICS), with patients most commonly escalating from LABA/ICS in the US and LAMA monotherapy in the UK. These analyses used data from large medical databases in the US and the UK that are representative of the COPD population in each country: MarketScan ${ }^{\circledR}$ includes data from 155.8 million patients across all regions in the US, and CPRD GOLD includes data from 14.7 million patients registered at $714 \mathrm{GP}$ practices across the UK. In the US, pharmacy dispensing information is seen as the gold standard of drug exposure information [30]. However, US prescribing patterns may be affected by factors such as drug formularies and respective insurance coverage and co-pays, which may influence practitioners to prescribe combination inhalers from the outset rather than stepping up therapy. Medicaid, for instance, limits the number of reimbursable prescriptions for branded medications [31]. In addition, prescriptions in the US may be influenced by treatment costs and formulary coverage.

A number of studies have found that adherence to national guidelines is associated with lower healthcare costs [32, 33]. A study using the Geisinger Health System database in the US identified patients initiating a first prescription for COPD and classified them as adherent or non-adherent to GOLD recommendations, based on their pulmonary function test, i.e., GOLD 1-4 [32]. Adhering to GOLD recommendations was associated with lower costs in patients with moderate to severe COPD [32]. Another study in the UK concluded that the treatment algorithm recommended by NICE in the UK represented a cost-effective approach for the management of COPD [33].

\section{Limitations}

This retrospective cohort study was conducted using data from analyzed longitudinal electronic healthcare records and is therefore subject to a number of limitations. While the records from MarketScan ${ }^{\circledR}$ and CPRD GOLD strive for high-quality data, it is possible that some patients with COPD and asthma may have had their condition incorrectly diagnosed/coded, influencing the prescribing patterns we observed (patients with asthma are more likely to be prescribed ICS). In addition, even for patients whose condition was correctly coded, individual values for demographic and clinical parameters may also have been missing from the database or recorded incorrectly. This is more likely to be a factor within the US dataset, given the well-documented high turnover rate of patients within US commercial datasets (e.g. due to changes in employment or marital status). MarketScan ${ }^{\circledR}$ data are provided by large employers and health plans, but it is possible that data from medium and small employers may be under-represented. CPRD GOLD only includes data from primary care, and although prescriptions from secondary settings are very uncommon, these may have been missed. Unlike the US database, the CPRD 
database records medications prescribed, but not medications dispensed.

Patients were required not to have used any maintenance medications in the year before cohort entry. Furthermore, maintenance therapies used for $<30$ days were excluded by design; therefore, patients with short overlaps of otherwise legitimate maintenance therapy episodes would be counted as not having any maintenance therapy use. As lung function data were not available in the MarketScan ${ }^{\circledR}$ database, it is not possible to know what proportion of US patients had a diagnosis of COPD based on airflow obstruction $\left(\mathrm{FEV}_{1} / \mathrm{FVC}<70 \%\right.$, as per the GOLD criteria). In a previous US study conducted in a community-based practice, only $43 \%$ of patients met the GOLD criterion for airflow obstruction [34]. This might partly explain the high use of LABA/ICS observed in our US dataset because, in the absence of pulmonary function data, physicians may be more inclined to prescribe ICS due to uncertainty around a potential diagnosis of asthma. The absence of spirometryconfirmed COPD in the US makes the overprescription we observed even more striking, as it suggests that many patients may have been prescribed ICS without confirmation of airflow obstruction. A further possibility is that discount coupons/rebates issued by drug manufacturers in the US, or prescribing restrictions for first-line therapy imposed by certain insurers, may have influenced prescribing patterns. In the UK dataset, not all data on hospitalizations or emergency department visits may be recorded in CPRD, which is a limitation because exacerbations are likely to be under-reported when no linkage to hospital data is performed. Lastly, exacerbation severity (moderate vs. severe) was not recorded in either country.

\section{CONCLUSIONS}

In this retrospective analysis of healthcare databases in the US and UK, $50.9 \%$ of US patients and $32.4 \%$ of UK patients had $\geq 1$ exacerbation in the year prior to initiating COPD maintenance therapy. In the US, twothirds of patients received ICS-containing therapies at 1MT, with almost half prescribed LABA/
ICS. In contrast, less than one-third received ICS-containing therapy in primary care in the UK, and more than half of patients received LAMA.

In both countries, more COPD patients (without an asthma co-diagnosis) received ICScontaining therapies at initiation of 1MT than would be expected based on their exacerbation history alone, indicating overprescription of ICS, particularly in the US. In the UK, patients were treated according to the 2010-2018 NICE guidelines and received LAMA at initiation of $1 \mathrm{MT}$, based on their history of exacerbations.

\section{ACKNOWLEDGEMENTS}

The authors wish to thank Christina Raabe of Boehringer Ingelheim for her contributions to data analyses and her insightful comments during manuscript review, as well as Edward Yu, Jocelyn Wang and Gandarvaka Miles of Aetion Inc., contracted by Boehringer Ingelheim, for their contributions to the protocol development and analytic implementation.

Funding. This analysis and the journal's Rapid Service Fee were funded by Boehringer Ingelheim.

Medical Writing and Editorial Assistance. Medical writing assistance, in the form of preparing and revising the manuscript, was funded by Boehringer Ingelheim and provided by Cindy Macpherson via MediTech Media, under the authors' conceptual direction and based on feedback from the authors.

Authorship. All named authors meet the International Committee of Medical Journal Editors (ICMJE) criteria for authorship for this article, take responsibility for the integrity of the work as a whole, and have given their approval for this version to be published.

Author Contributions. JM contributed substantially to the design of the analysis; EMG (as part of Aetion Inc.) provided the data. All authors contributed to the analysis of the data, 
as well as to the drafting and revision of the manuscript.

Disclosures. Chloe I. Bloom has nothing to disclose. Olaf Jöns is a full-time employee of Boehringer Ingelheim, and Jukka Montonen was a full-time employee of Boehringer Ingelheim at the time of study conduct (current affiliation: Merck KGaA, Darmstadt, Germany). Elizabeth M. Garry is a full-time employee of Aetion, Inc., with stock options. Surya P. Bhatt has received consulting fees from Boehringer Ingelheim and Sanofi, and participated in advisory boards for Sunovion and GlaxoSmithKline. In addition, his academic institute has received research grants from Sanofi and the National Institutes of Health.

Compliance with Ethics Guidelines. As this was an observational study based on de-identified patient-level data from healthcare databases reported in aggregate only, the study did not require ethics committee approval, nor were subjects required to provide informed consent.

Data Availability. The data for this analysis were provided by Aetion Inc. under license and are therefore not publicly available. Upon reasonable request, access to the data can be provided by the corresponding author, with the permission of Aetion Inc.

Open Access. This article is licensed under a Creative Commons Attribution-NonCommercial 4.0 International License, which permits any non-commercial use, sharing, adaptation, distribution and reproduction in any medium or format, as long as you give appropriate credit to the original author(s) and the source, provide a link to the Creative Commons licence, and indicate if changes were made. The images or other third party material in this article are included in the article's Creative Commons licence, unless indicated otherwise in a credit line to the material. If material is not included in the article's Creative Commons licence and your intended use is not permitted by statutory regulation or exceeds the permitted use, you will need to obtain permission directly from the copyright holder. To view a copy of this licence, visit http://creativecommons.org/licenses/by$\mathrm{nc} / 4.0 /$.

\section{REFERENCES}

1. May SM, Li JT. Burden of chronic obstructive pulmonary disease: healthcare costs and beyond. Allergy Asthma Proc. 2015;36(1):4-10.

2. Kochanek $\mathrm{KD}, \mathrm{Xu} \mathrm{JQ}$, Arias E. Mortality in the United States, 2019. 2020. https://www.cdc.gov/ nchs/products/databriefs/db395.htm. Accessed 29 Jan 2021.

3. Snell N, Strachan D, Hubbard R, Gibson J, GruffyddJones K, Jarrold I. S32 Epidemiology of chronic obstructive pulmonary disease (COPD) in the UK: findings from the British Lung Foundation 'respiratory health of the nation' project. Thorax. 2016;71(Suppl 3):A20.1.

4. American Lung Association. Learn about COPD. 2020. https://www.lung.org/lung-health-diseases/ lung-disease-lookup/copd/learn-about-copd. Accessed 14 May 2020.

5. Guarascio AJ, Ray SM, Finch CK, Self TH. The clinical and economic burden of chronic obstructive pulmonary disease in the USA. Clinicoecon Outcomes Res. 2013;5:235-45.

6. Ford ES, Murphy LB, Khavjou O, Giles WH, Holt JB, Croft JB. Total and state-specific medical and absenteeism costs of COPD among adults aged $>/=$ 18 years in the United States for 2010 and projections through 2020. Chest. 2015;147(1):31-45.

7. British Lung Foundation. Chronic obstructive pulmonary disease (COPD) statistics. https://statistics. blf.org.uk/copd. Accessed 29 Jan 2021.

8. The Lancet. UK COPD treatment: failing to progress. Lancet. 2018;391(10130):1550.

9. British Lung Foundation. The battle for breath - the economic burden of lung disease. 2020. https:// www.blf.org.uk/policy/economic-burden. Accessed 25 Sept 2020.

10. Global Initiative for Chronic Obstructive Lung Disease. Global strategy for the diagnosis, management, and prevention of chronic obstructive pulmonary disease (2021 report). 2020. https:// goldcopd.org/wp-content/uploads/2020/11/GOLDREPORT-2021-v1.1-25Nov20_WMV.pdf. Accessed 02 Feb 2021. 
11. Nici L, Mammen MJ, Charbek E, Alexander PE, Au $\mathrm{DH}$, Boyd CM, et al. Pharmacologic management of chronic obstructive pulmonary disease. An official American Thoracic Society Clinical Practice Guideline. Am J Respir Crit Care Med. 2020;201(9): e56-69.

12. National Institute for Health and Care Excellence. Chronic obstructive pulmonary disease in over 16s: diagnosis and management. 2018. https://www. nice.org.uk/guidance/ng115/resources/chronicobstructive-pulmonary-disease-in-over-16sdiagnosis-and-management-pdf-66141600098245. Accessed 29 Apr 2021.

13. Arnold MJ. Treatment of chronic obstructive pulmonary disease: guidelines from the American Thoracic Society. Am Fam Physician. 2021;104(1): 102-3.

14. Barrecheguren M, Monteagudo M, Ferrer J, Borrell E, Llor C, Esquinas C, et al. Treatment patterns in COPD patients newly diagnosed in primary care. A population-based study. Respir Med. 2016;111:47-53.

15. Diette GB, Dalal AA, D'Souza AO, Lunacsek OE, Nagar SP. Treatment patterns of chronic obstructive pulmonary disease in employed adults in the United States. Int J Chron Obstruct Pulmon Dis. 2015;10:415-22.

16. Chalmers JD, Tebboth A, Gayle A, Ternouth A, Ramscar N. Determinants of initial inhaled corticosteroid use in patients with GOLD A/B COPD: a retrospective study of UK general practice. NPJ Prim Care Respir Med. 2017;27(1):43.

17. Bloom CI, Elkin SL, Quint JK. Changes in COPD inhaler prescriptions in the United Kingdom, 2000 to 2016. Int J Chron Obstruct Pulmon Dis. 2019;14: 279-87.

18. Suissa S, Kezouh A, Ernst P. Inhaled corticosteroids and the risks of diabetes onset and progression. Am J Med. 2010;123(11):1001-6.

19. Suissa S, Dell'Aniello S, Ernst P. Comparative effectiveness and safety of LABA-LAMA vs LABA-ICS treatment of COPD in real-world clinical practice. Chest. 2019;155(6):1158-65.

20. Price D, Yawn B, Brusselle G, Rossi A. Risk-to-benefit ratio of inhaled corticosteroids in patients with COPD. Prim Care Respir J. 2013;22(1):92-100.

21. Dahl R. Systemic side effects of inhaled corticosteroids in patients with asthma. Respir Med. 2006;100(8):1307-17.

22. Herrett E, Gallagher AM, Bhaskaran K, Forbes H, Mathur R, van Staa T, et al. Data resource profile: clinical practice research datalink (CPRD). Int J Epidemiol. 2015;44(3):827-36.

23. Garcia Rodriguez LA, Perez GS. Use of the UK general practice research database for pharmacoepidemiology. Br J Clin Pharmacol. 1998;45(5): 419-25.

24. Walley T, Mantgani A. The UK general practice research database. Lancet. 1997;350(9084):1097-9.

25. Rothnie KJ, Mullerova H, Thomas SL, Chandan JS, Smeeth L, Hurst JR, et al. Recording of hospitalizations for acute exacerbations of COPD in UK electronic health care records. Clin Epidemiol. 2016;8: 771-82.

26. Rothnie KJ, Mullerova H, Hurst JR, Smeeth L, Davis $\mathrm{K}$, Thomas SL, et al. Validation of the recording of acute exacerbations of COPD in UK primary care electronic healthcare records. PLOS ONE. 2016;11(3): e0151357.

27. Wang S, Verpillat P, Rassen J, Patrick A, Garry E, Bartels D. Transparency and reproducibility of observational cohort studies using large healthcare databases. Clin Pharmacol Ther. 2016;99(3): 325-32.

28. NICE National Clinical Guideline Centre. Chronic obstructive pulmonary disease in over 16s: diagnosis and management. 2010. https://www.nice.org. uk/guidance/CG101. Accessed 06 Nov 2010.

29. Gruffydd-Jones K, Loveridge C. The 2010 NICE COPD guidelines: how do they compare with the GOLD guidelines? Prim Care Respir J. 2011;20(2): 199-204.

30. West SL, Savitz DA, Koch G, Strom BL, Guess HA, Hartzema A. Recall accuracy for prescription medications: self-report compared with database information. Am J Epidemiol. 1995;142(10):1103-12.

31. Lieberman DA, Polinski JM, Choudhry NK, Avorn J, Fischer MA. Medicaid prescription limits: policy trends and comparative impact on utilization. BMC Health Serv Res. 2016;16:15.

32. Asche CV, Leader S, Plauschinat C, Raparla S, Yan $\mathrm{M}$, Ye $\mathrm{X}$, et al. Adherence to current guidelines for chronic obstructive pulmonary disease (COPD) among patients treated with combination of longacting bronchodilators or inhaled corticosteroids. Int J Chronic Obstruct Pulm Dis. 2012;7:201-9.

33. Hertel N, Kotchie RW, Samyshkin Y, Radford M, Humphreys S, Jameson K. Cost-effectiveness of available treatment options for patients suffering from severe COPD in the UK: a fully incremental analysis. Int J Chronic Obstruct Pulm Dis. 2012;7: 183-99. 
34. Alabi FO, Alkhateeb HA, DeBarros KM, Barletti Benel PS, Sanchez-Martez RL, Zeper ML, et al. The heterogeneity of COPD patients in a communitybased practice and the inadequacy of the global initiative for chronic obstructive lung disease criteria: a real-world experience. Chronic Obstr Pulm Dis. 2021;8(3):396-407. 